\title{
The Effect of Proton Damage on the X-Ray Spectral Response of MOS CCDs for the Swift X-ray Telescope
}

\author{
R. M. Ambrosi ${ }^{\dagger}$, A. D. T. Short, A. F. Abbey, A. A. Wells \& D. R. Smith \\ X-ray Astronomy Group, Department of Physics and Astronomy, Leicester University, Leicester, UK
}

\begin{abstract}
The effect of non-ionising energy loss (NIEL) of protons in charge-coupled devices is to displace silicon atoms and any dopant materials present from their lattice positions to form lattice defects which in turn can trap electrons [1]. A CCD operating as a photon counter for x-ray spectroscopy relies on the efficient transfer of charge from one region to another. The number of defects produced will reduce the charge transfer efficiency (CTE) and hence degrade the spectral resolution of the energy distribution of interest [2]. The Swift X-ray Telescope will be equipped with a single EPIC MOS CCD22 as developed for the XMM project [3]. It is the aim of this study to determine the effect of the radiation environment on the performance of the CCD and its impact on the scientific objective of the x-ray telescope, to probe the x-ray afterglow of Gamma Ray Bursts (GRBs).
\end{abstract}

Keywords: Charge-coupled device, radiation, damage, x-ray, spectroscopy, resolution.

PACS: 95.55.A, 95.85.N, 07.89

\section{Introduction}

The Swift X-ray Telescope (XRT) is part of the Swift Gamma Ray Burst Explorer, which is a three-telescope space observatory for studying the position, brightness, and physical properties of gamma ray bursts $[4,5]$.

${ }^{\dagger}$ Corresponding author: R. M. Ambrosi, University of Leicester, Department of Physics and Astronomy, Space Research Centre, University Rd, Leicester LE1 7RH, UK, telephone: +44 (0) 116 2231812, fax: +44 (0) 116 2522464, e-mail: rma@star.le.ac.uk 
The XRT is a sensitive, flexible, autonomous X-ray CCD imaging spectrometer designed to measure the position, spectrum, and brightness of gamma-ray bursts (GRBs) and afterglows over a wide dynamic range covering more than seven orders of magnitude in flux [4, 5]. An x-ray spectroscopy capability enables the determination of the redshift of the GRB if any spectral lines are visible in the x-ray afterglow [4, 5]. The required spectral resolution is $300 \mathrm{eV}$ at $6.4 \mathrm{keV}$ for the three year mission lifetime [4, 5].

In order to accomplish this, the x-ray response of the CCD as a function of non-ionising damage must be evaluated. The proton induced displacement of silicon atoms from their lattice positions and the formation of inter-band charge traps is well-documented [1]. The density of traps is proportional to absorbed proton fluence and gives a corresponding reduction in CTE and hence spectral resolution[2].

\section{Radiation Environment}

Swift will be placed in a $600 \mathrm{~km}, 22^{\circ}$ orbit. It will periodically traverse the South Atlantic Anomaly and will be exposed to a hard spectrum of trapped protons with incident flux ranging between 100 and 1000 protons. $\mathrm{cm}^{-2} \cdot \mathrm{s}^{-1}$ (See Figure 1) [6].

In this study all the modelling of the space environment was accomplished with the Space Environment Information System (SPENVIS) Monte Carlo code [6].

The shield for the CCD was modelled as a spherical aluminium shell. A spherical shell is an accurate approximation of the actual shield, which has been designed in such a way that the CCD is surrounded by equal thicknesses of aluminium in all directions [6]. Figure 2 shows a section through shield used in the model [6].

At an altitude of $600 \mathrm{~km}$, the damage caused by soft protons reaching the CCD as a result of grazing incidence reflection through the mirror optics is negligible [6]. 
Figure 3 shows how the proton fluence at the centre of a spherical aluminium shell varies as a function of spherical shell thickness. For this study a limiting shielding thickness of $60 \mathrm{~mm}$ was chosen since a thicker aluminium shield would not significantly reduce the dose to the CCD, whilst any further increase in mass would place a strain on the mechanical design. Table 1 reflects the total dose that the CCD will receive (over a period of three years) if the shield is modelled as in Figure 2 [6].

From the data in Table 1, the CCD would be exposed to a total $10 \mathrm{MeV}$ equivalent proton dose of $7.3 \times 10^{8}$ protons. $\mathrm{cm}^{-2}$ during the initial three years of the Swift mission. Although this is a conservative estimate the equivalent proton dose is not expected to exceed $1.0 \times 10^{9}$ protons.cm ${ }^{-2}$. It is therefore important that this radiation damage study be carried out for a total dose of $1.0 \times 10^{9}$ protons. $\mathrm{cm}^{-2}$ and that the resolution target of $300 \mathrm{eV}$ for $6.4 \mathrm{keV}$ x-rays be met at the end of life.

\section{X-ray Spectroscopy with the EPIC MOS CCDs}

X-ray spectroscopy with CCDs is possible when they are operated as photon counters. A low noise output from the device, high isolated event detection efficiency and a good charge transfer efficiency (CTE) are prerequisites for photon counting with CCDs [7]. The resolution of an energy distribution is measured as the full width at half maximum (FWHM) [7]. The resolution is directly dependent on those properties of the detection medium that affect the generation, transport, and collection of charge and noise contributions from the external electronics [7].

\subsection{Peak Broadening}

The mean readout noise is of the order of 4 electrons rms at $-100^{\circ} \mathrm{C}$ for the CCD22s and test electronics that were used in this study. The variance of the normal distribution can be described by $\sigma_{r}^{2}$. Other noise components include the carrier generation noise also termed Fano noise. If carrier generation were purely statistical, then [7]: 
$\sigma^{2}=N=\mu=\frac{E_{0}}{\omega(E)}$

where $\mu$ is the mean number of carriers, $E_{0}$ is the incident x-ray energy, $\omega$ is the energy required to produce an electron-hole pair. Since carrier generation is not purely statistical, the variance is given by [7]:

$\sigma_{g}^{2}=\frac{F E_{0}}{\omega} \quad$ where $\quad \sigma_{g}^{2}<(N=\mu)$ and $\quad F<1$

where $F$ is the Fano factor. Dark current noise, with variance $\sigma_{d}^{2}$, can be regarded as negligible at the operating temperatures of these devices.

The EEV CCD22s are front illuminated frame transfer devices consisting of $600 \mathrm{x} 600,40 \mu \mathrm{m}$ square pixels in the image section and $600 \times 602,39 \times 12 \mu \mathrm{m}$ pixels in the store section [3]. Charge transfer noise is the contribution due to charge losses during the transfer of charge along the parallel and horizontal shift register of the CCD. The variance on the noise contribution from the CTE in the parallel and serial registers is summarised by [7]:

$\sigma_{t}^{2}=2 \frac{E_{0}}{\omega}\left[\left(1-C T E_{p}^{N t p}\right)+C T E_{p}^{N t p}\left(1-C T E_{s}^{N t s}\right)\right]$

where $C T E_{p}$ is the charge transfer efficiency in the parallel register, $C T E_{s}$ the charge transfer efficiency in the serial register and $N t$ the number of transfers either in the parallel or the serial registers. In addition to the noise contribution from charge losses during transfer, the observed reduction in resolution is also effected by a shift in the photopeak for a broad illumination of the CCD.

Given all these sources of noise, the broadened spectral line can be described by [7]: 
$n(E)=\frac{n_{0}}{\sqrt{2 \pi \sigma}} \exp \left[-\frac{\left(E-E_{0}\right)^{2}}{2 \sigma^{2}}\right]$

where $n(E)$ is the broadened spectral line, $n_{0}$ is the infinitely sharp line, $E$ is the energy and $\sigma^{2}$ the variance, where $\sigma^{2}$ is also [7]:

$\sigma^{2}=\sigma_{g}^{2}+\sigma_{r}^{2}+\sigma_{d}^{2}+\sigma_{t}^{2}$

\subsection{Scientific Implications}

A key point of interest for the $\mathrm{x}$-ray astronomer is the resolution of the 6-7 keV iron complex. Until recently there has been some doubt as to whether or not iron lines will feature in GRB x-ray spectra. This depends on the nature of the GRB progenitor. However, recently, Amati et al [8] have reported on redshifted iron absorption features in observations made by BeppoSax of GRB990705, whilst Piro et al [9] have reported on iron emission spectra in Chandra X-ray Observatory observations of GRB 991216.

\section{Radiation Hardness of EPIC-MOS CCDs}

The EPIC MOS EEV CCD22s used in this study were manufactured by Marconi Applied Technologies using Si-gate buried n-channel technology [10]. The same technique was used in the fabrication of the predecessors of these CCDs, the Jet-X CCD12s. Charge transferred through the parallel and the serial registers is confined to an implanted narrow supplementary buried channel [10]. Therefore any reduction in CTE due to radiation damage would be dependent on the size of the supplementary buried channel and hence the charge confinement area within each electrode [10]. The fact that the charge is confined to a supplementary buried channel of the order of $4 \mu \mathrm{m}$ in the CCD22s when compared to the $5 \mu \mathrm{m}$ for the Jet-X chips improves the overall radiation hardness of these chips [10]. The effective channel width of $4 \mu \mathrm{m}$ was achieved by including 
shallow surface-pinning implant on either side of the supplementary buried channel. Holmes-Siedle and Holland et al have described how the radiation hardness of the EPIC MOS CCDs will scale linearly with the total charge confinement area of the electrode and hence the width of the supplementary buried channel $[10$, 11]. Therefore for the case described above, the improvement in radiation hardness is approximately $20 \%$.

\section{Experimental Method}

The dedicated proton damage beam line at the tandem accelerator facility, run by AEA Technologies in Harwell, Didcot [12], was employed in this study. Figure 4 illustrates the beam line and the sample chamber. The $10 \mathrm{MeV}$ proton beam is extracted from the accelerator tube at $1 \mu \mathrm{A}$. The beam current is measured using an analysing magnet. Further down the beam line the focused proton beam is scattered by a series of scattering foils so that the protons are evenly distributed over the sample plate. By rotating the sample plate out of the beam, the charge accumulated in a Faraday cup and the number of scattered particles in the solid state detector are used to determine the number of particles reaching the sample plate. This calibration step is used to determine the exposure time required to give the sample the required proton dose. Since the doses required for this study were of the order of $5.0 \times 10^{8}$ protons. $\mathrm{cm}^{-2}$, the current was reduced to $400 \mathrm{nA}$ and a beam chopper was used to reduce it further to $1 \mathrm{nA}$. The CCD was mounted on the sample plate and rotated into the path of the scattered proton beam. Initially half the CCD was exposed to a fluence of $5.0 \times 10^{8}$ protons. $\mathrm{cm}^{-2}$, the other half was protected by a 3mm thick aluminium shield. Figure 5 shows a photograph of the CCD mounted on the sample plate, the aluminium proton shield is also visible. The entire CCD was then exposed to a second dose of $5.0 \times 10^{8}$ protons. $\mathrm{cm}^{-2}$. This produced two different regions of damage and hence two points on the resolution versus dose curve that would be extracted from the data. Another CCD from the same batch of devices had previously been exposed to a $2.7 \times 10^{8}$ protons.cm ${ }^{-2} 10 \mathrm{MeV}$ equivalent proton dose. The data from the two chips was used to produce an accurate assessment of how the CCD to be incorporated in the Swift X-ray telescope would degrade in the radiation environment discussed in Section 2. 
In a similar study, performed by Holmes-Sieldle and Holland , the predecessors of the EPIC CCDs, the Jet-X CCDs were used to determine how x-ray spectral resolution is affected by proton damage. In this previous study $6 \mathrm{keV}$ iron $\mathrm{x}$-rays were also used to predict how the CCD12s would perform as a function of proton damage. Since the radiation hardness scales with the charge confinement area, the ratio of the charge confinement areas for the two CCDs was used to scale the energy resolution of the $6 \mathrm{keV}$ spectral distributions obtained in the Jet-X study.

\section{The Physical Model}

Equations 1 to 5 were used to calculate the energy resolution of the iron photopeak as a function of proton dose. Charge transfer efficiency data obtained before and after proton damage were incorporated in the model. The contribution to line broadening from the shift in the photopeak was modelled by dividing a 500 frame deep CCD image into small regions of interest 100 pixel x 100 pixel square and determining the photopeak position for each of these regions. The number of parallel and serial transfers from the centres of each of these regions were used to determine the CTE contribution to the variance of the energy distribution. This combination of two effects was used to calculate the photopeak energy resolution.

\section{Results}

The variation in the energy resolution of the $6 \mathrm{keV}$ iron line as a function of radiation damage in Jet- $\mathrm{X}$ CCD12s is depicted in Figure 6. In the Swift radiation environment these chips would not be able to resolve the $6 \mathrm{keV}$ iron line to better than $300 \mathrm{eV}$ unless the temperature were maintained at $-110^{\circ} \mathrm{C}$.

By scaling the results obtained with the Jet-X chips with the ratio of the widths of the supplementary buried channels in CCD22s and CCD12s, an estimate of performance of the EPIC-CCDs as a function of radiation damage can be obtained. These estimates are depicted as a function of temperature in Figure 7. Included in 
Figure 7 are the physical model predictions of how the EPIC CCDs will perform with increasing radiation damage. Since the Swift x-ray telescope has been designed to maintain the CCD at an operating temperature of $-105^{\circ} \mathrm{C}$, the focus of this study has been the effect of radiation damage on an EPIC-MOS CCD operating at this temperature. From the graph depicted in Figure 7 there is very good agreement between the predictions made from the Jet-X data for CCD22s operating at $-105^{\circ} \mathrm{C}$ and the physical model description of the performance of the EPIC CCD at this temperature. At $-90^{\circ} \mathrm{C}$, the predictions described by Figure 7 show that CCD22s will just miss the $300 \mathrm{eV}$ requirement at the end of the three year mission but only by $12 \mathrm{eV}$, or less than $5 \%$.

The experimental results obtained by irradiating an EPIC-MOS CCD with $10 \mathrm{MeV}$ protons at Harwell are shown in Figure 8. Although not as good as the predictions described in Figure 7, the model also shows good aggreement with the experimental data. According to the experimental results even at $-90^{\circ} \mathrm{C}$ the EPIC CCDs should meet the post three year energy resolution target of $300 \mathrm{eV}$.

\section{Conclusions}

The results obtained in this study confirm that a reduction in the width of the narrow buried channel from CCD12s to CCD22s increased the radiation hardness of the CCD. The predicted performance of CCD22s as a function of radiation damage (based on Jet-X data) agreed within experimental errors. The predicted resolution values for doses of $1.0 \times 10^{9}$ protons.cm ${ }^{-2}$ differ from the experimental results by only $7 \%$. This further substantiates the approximation that radiation hardness and hence energy resolution scales as the ratio of the widths of the supplementary buried channels in CCDs fabricated by Si-gate buried n-channel technology.

Although the initial results obtained with Jet-X CCDs indicated that the baseline resolution of $300 \mathrm{eV}$ would not be met 3 years after launch, the experimental results showed that the EPIC-MOS CCD22s would meet this energy resolution target at temperatures ranging between $-90^{\circ} \mathrm{C}$ and $-110^{\circ} \mathrm{C}$. The results inmply that if the 
proton shield thickness is kept unchanged at $6 \mathrm{~cm}$, the operating temperature of the CCD can be increased by $5^{\circ} \mathrm{C}$ to $10^{\circ} \mathrm{C}$ without compromising the mission requirements. This represents an appropriate margin in the thermo-mechanical design of the X-ray telescope.

\section{Acknowledgements}

The authors would like to thank Dr. Keith Jones and Mr. Ray Johnson at Harwell, Didcot, for their assistance during the experimental phase of this study.

\section{References}

[1] C. Dale, P. Marshall, B. Cummings, L. Shamey, A. Holland, IEEE Trans. Nucl. Sci. NS-40 (1993) 1628.

[2] A. D. Holland, The effect of bulk traps in proton irradiated EEV CCD's, Jet-X Project Document: JET-X(94)UL-230 WP2220 (1994).

[3] A. D. Short, A. Keay, M. J. L. Turner, SPIE 3445 (1998) 13.

[4] N. Gehrels, Swift gamma-ray burst MIDEX mission, SPIE 4140 (2000) 42.

[5] D. N. Burrows, J. E. Hill, J. A. Nousek, A. A. Wells, A. D. Short, R. Willingale, O. Citterio, G. Chincarini, G. Tagliaferri, S. Campana, Swift x-ray telescope, SPIE 4140 (2000) 64.

[6] A. D. Short, Swift XRT: proton environment and CCD degradation, Swift Project Document: SWIFTLUX-RE-001/0 (2000).

[7] A. Owens, K. J. McCarthy, Spectral degradation effects in X-ray CCD’s, Jet-X Project Document: JET-X(94)UL-230 WP2220 (1994).

[8] L. Amati, F. Frontera, M. Vietri, et al, Science 290 (2000) 953.

[9] L. Piro, G. Garmire, M. Garcia, et al, Science 290 (2000) 955. 
[10] S. Watts, A. Holmes-Siedle, A. Holland, Further Radiation Evaluation of X-ray Sensitive Charge Coupled Devices (CCDs) for the XMM Telescope, Brunel University Report BRUCRD-ESACCD-951R (1995).

[11] A. Holmes-Siedle, A. Holland, S. Watts, Brunel University Report BRUCRD-NSREC-96-1R (1996).

[12] G. R. Hopkinson, Proton induced Changes in CTE for n-channel CCD's and the effect on star tracker performance, IEEE Trans. Nucl. Sci. (In Print). 
Table 1 . The contributions to the overall $10 \mathrm{MeV}$ equivalent proton dose of $7.3 \times 10^{8}$ protons.cm ${ }^{-2}$ received by the CCD over a three year period.

\begin{tabular}{|c|c|c|c|c|}
\hline $\begin{array}{l}\text { Aluminium Shield } \\
\text { Thickness (mm) }\end{array}$ & $\begin{array}{l}10 \mathrm{MeV} \text { proton fluence } \\
\text { (fluence. } 4 \pi^{-1} \cdot 3 \mathrm{yr}^{-1} \text { ) }\end{array}$ & $\begin{array}{c}\text { Solid Angle } \\
\text { (sr) }\end{array}$ & $\mathrm{sr} / 4 \pi$ & $\begin{array}{l}\text { Net Contribution } \\
\left(\mathrm{p} \cdot \mathrm{cm}^{-2} \cdot 3 \mathrm{yr}^{-1}\right)\end{array}$ \\
\hline 0 & $6.2 \times 10^{9}$ & $5.7 \times 10^{-5}$ & $4.5 \times 10^{-6}$ & $2.8 \times 10^{4}$ \\
\hline 1 & $1.9 \times 10^{9}$ & 0.15 & $1.2 \times 10^{-2}$ & $2.1 \times 10^{7}$ \\
\hline 60 & $7.1 \times 10^{8}$ & 12.43 & $9.9 \times 10^{-1}$ & $7.1 \times 10^{8}$ \\
\hline
\end{tabular}




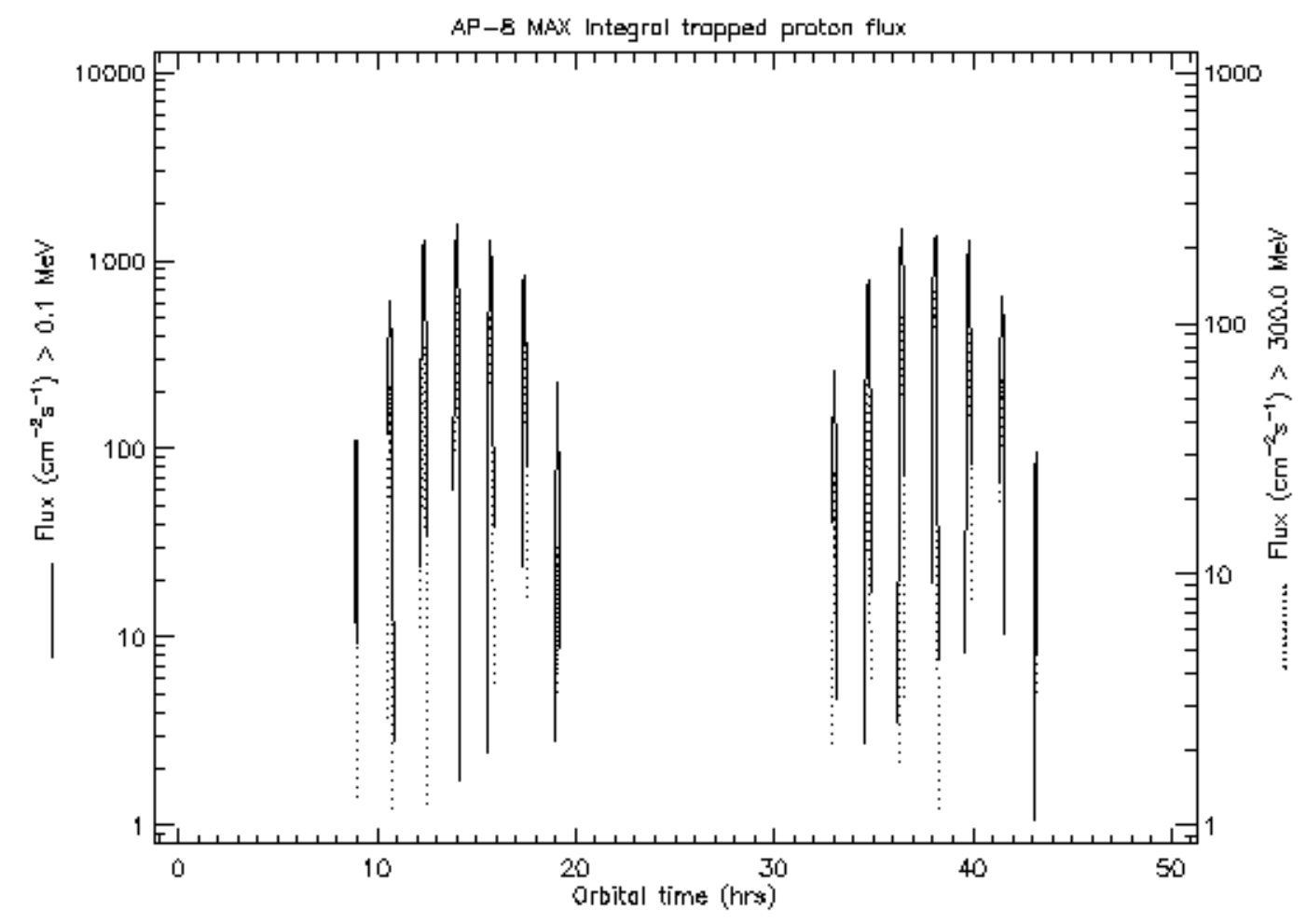

Figure 1 


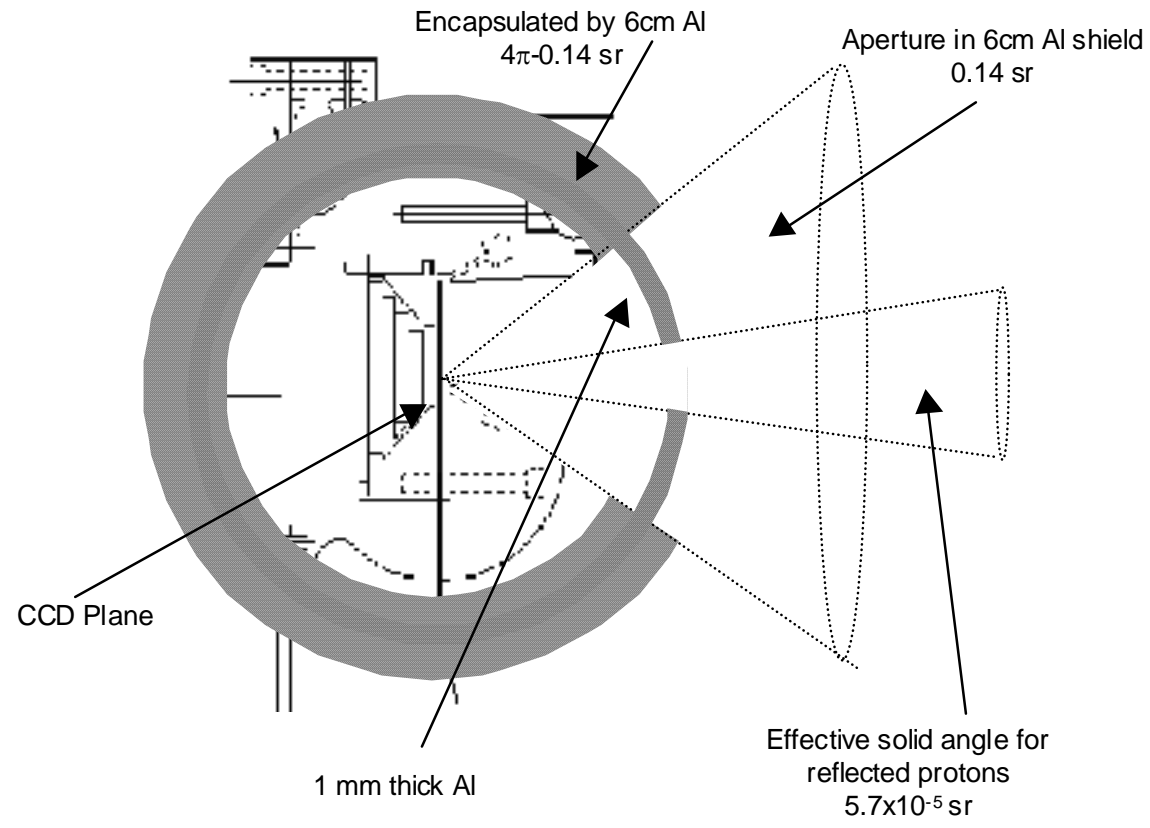

Figure 2 


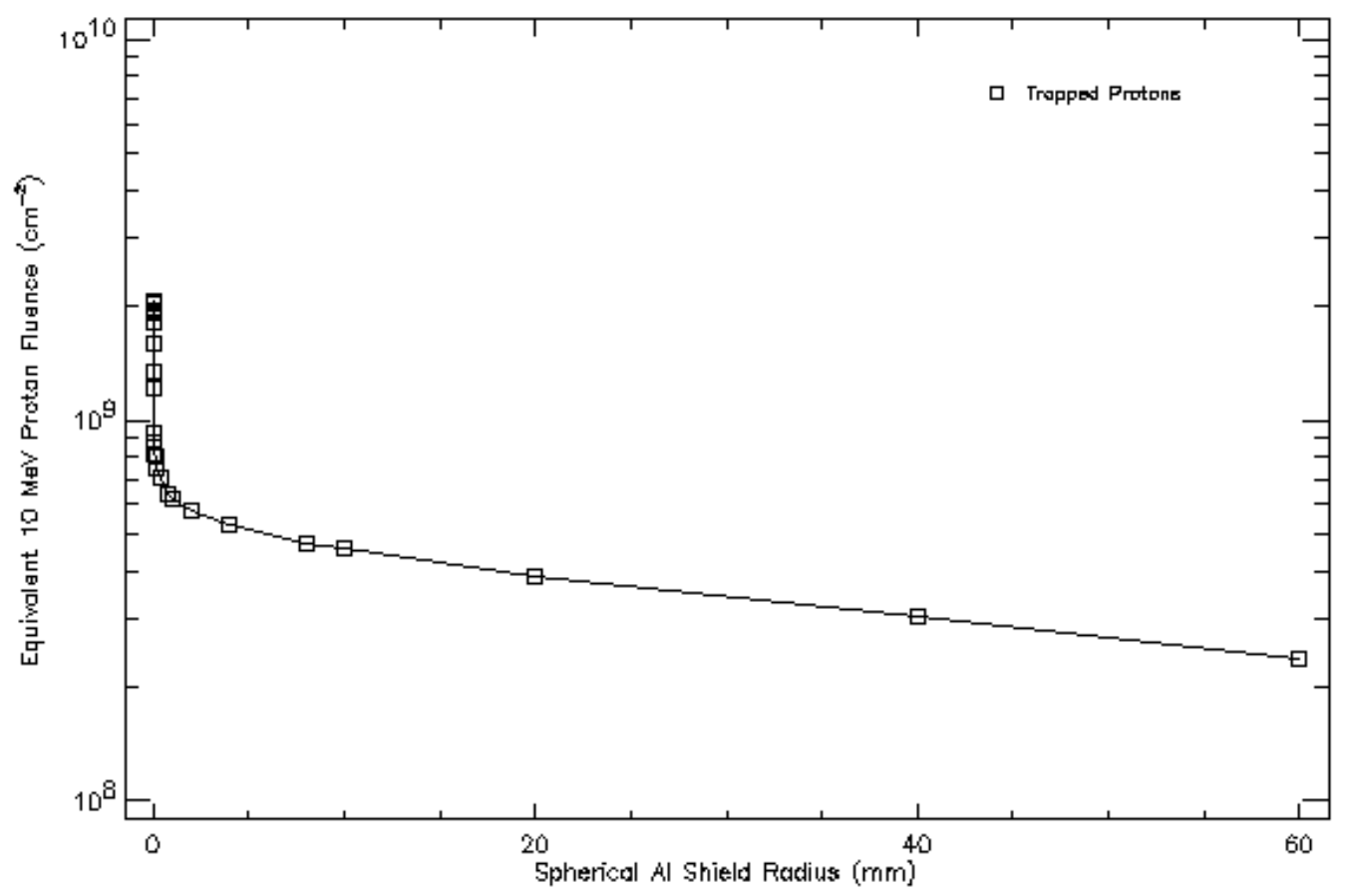

Figure 3 


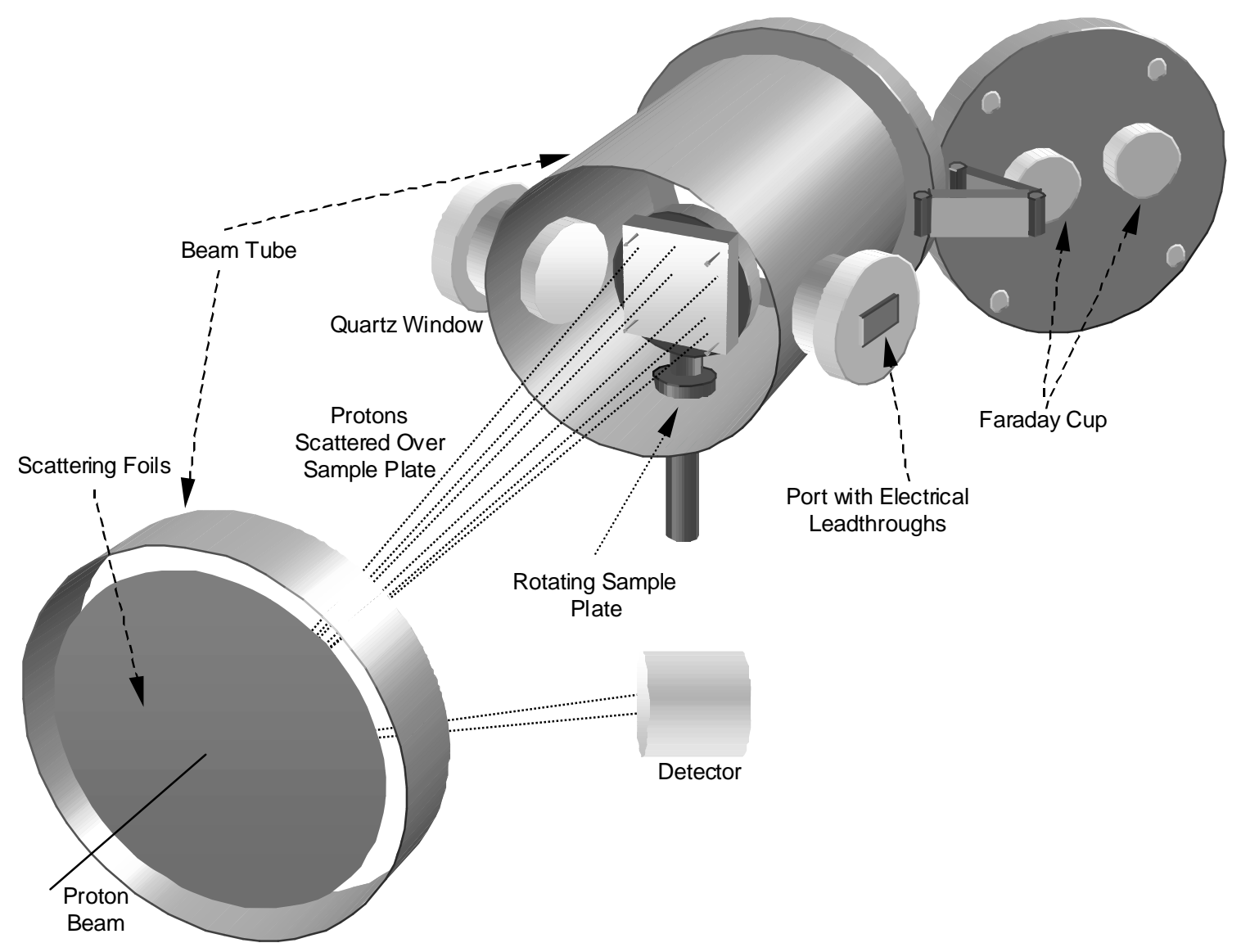

Figure 4 


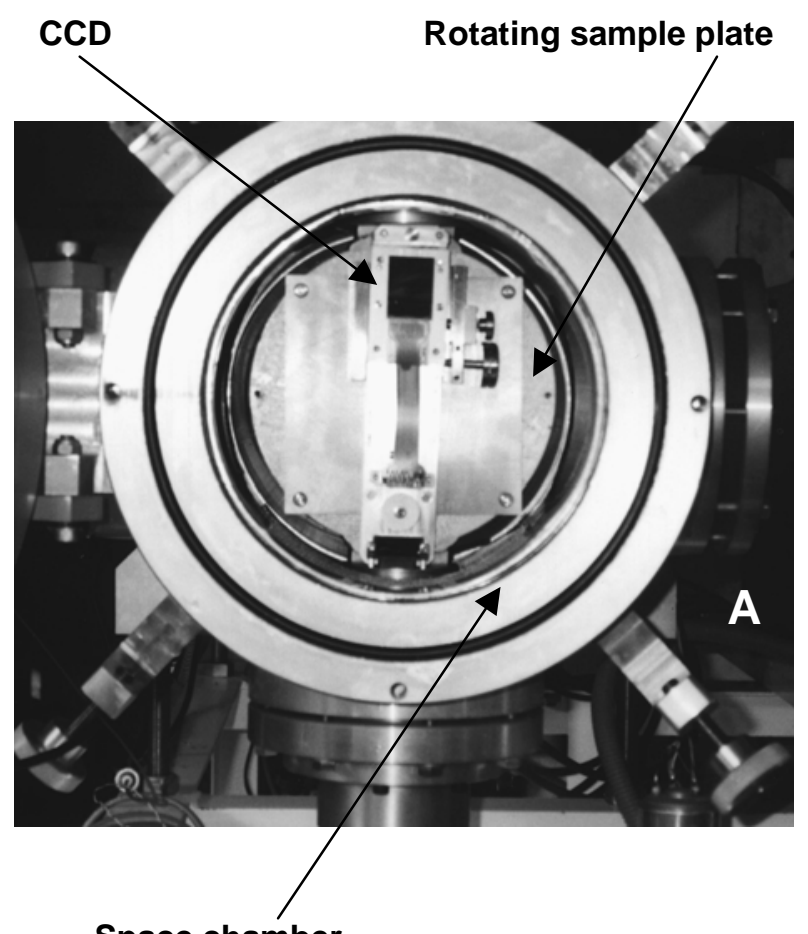

Space chamber

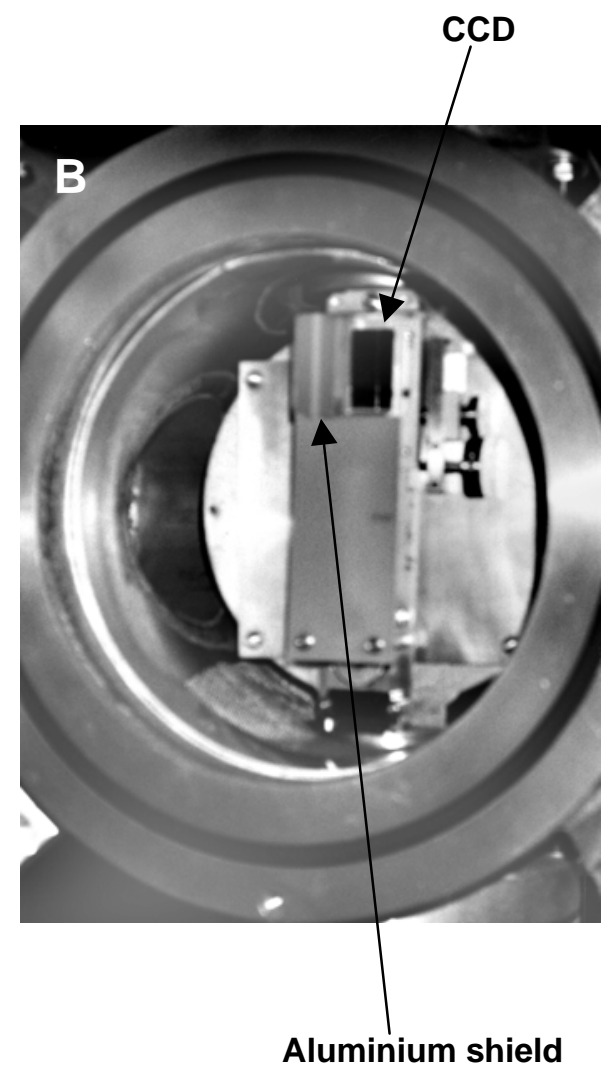

Figure 5 


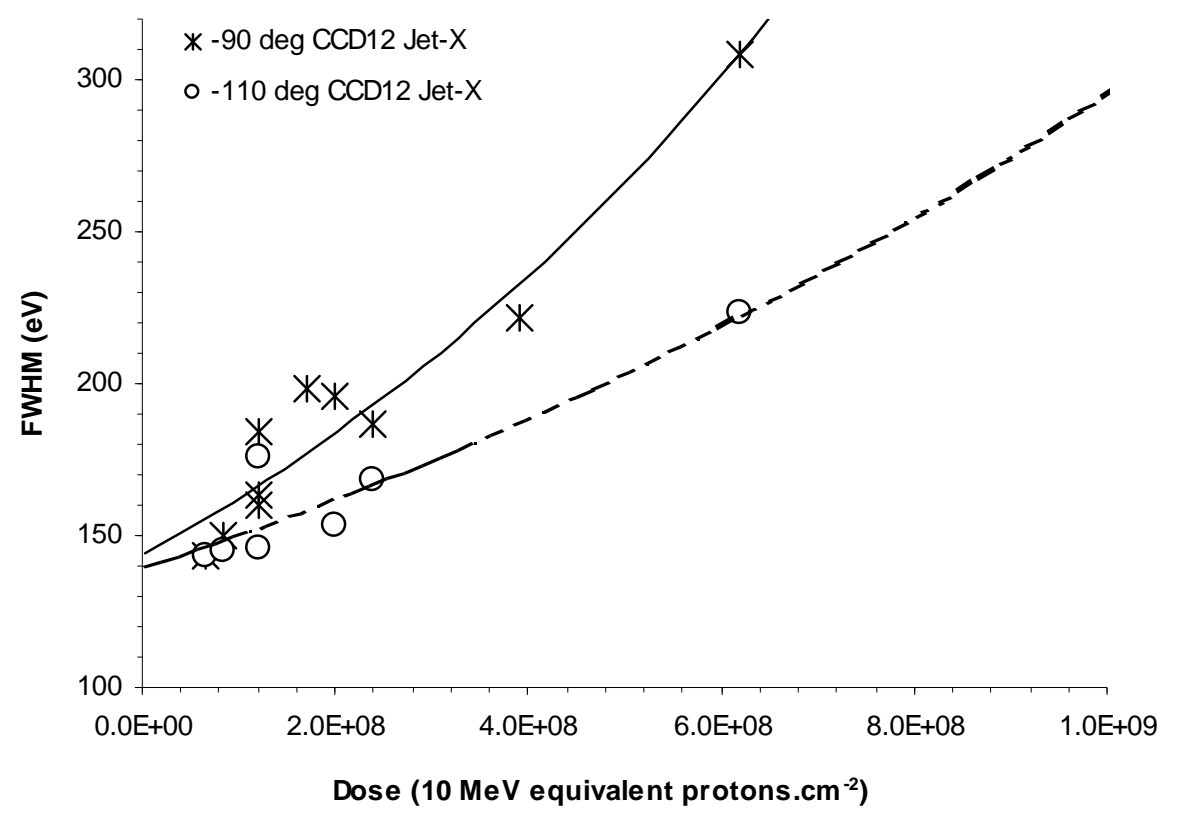

Figure 6 


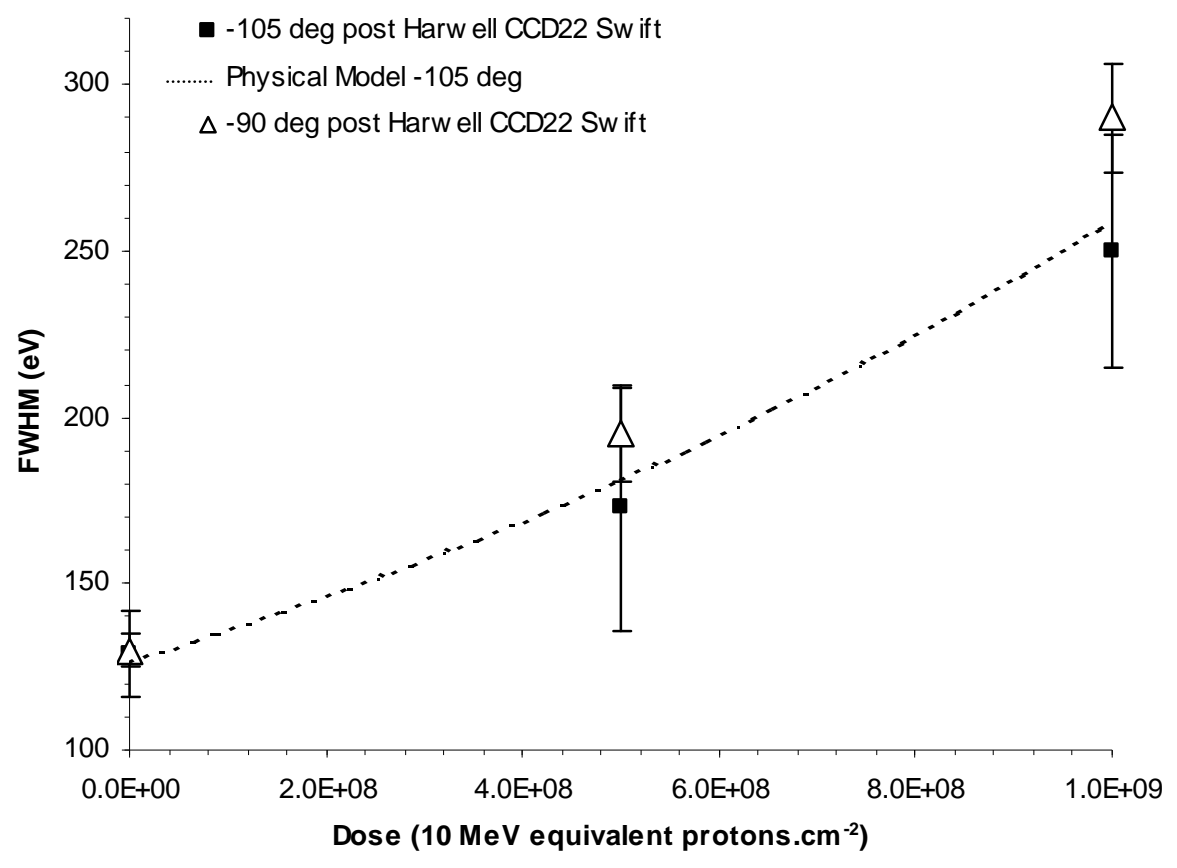

Figure 7

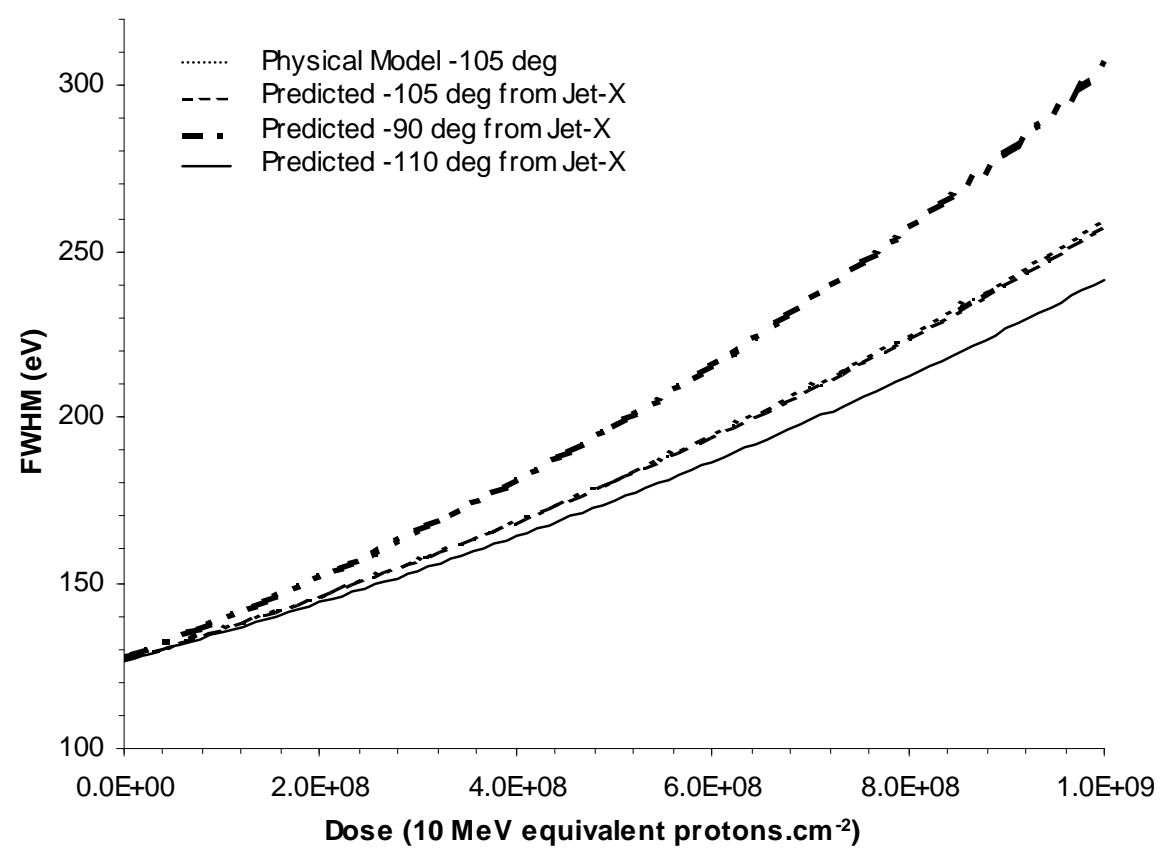

Figure 8 
Figure 1. Proton flux as a function of time for a $600 \mathrm{~km} 22^{\circ}$ orbit [6].

Figure 2. Cross section through the model of a spherical shield. The CCD is almost completely surrounded by $6 \mathrm{~cm}$ of aluminium.

Figure 3. One year proton fluence at the CCD for the spacecraft in a $600 \mathrm{~km} 22^{\circ}$ orbit as a function of the thickness of a spherical aluminium shell [6].

Figure 4. Illustration of the proton beam line and space chamber at the tandem accelerator facility at Harwell, Didcot.

Figure 5. (A) A CCD mounted onto the sample plate in the space chamber on the proton beam line at Harwell. (B) The $3 \mathrm{~mm}$ thick proton shield used to protect one half of the CCD is visible.

Figure 6. The variation in energy resolution of $6 \mathrm{keV}$-rays as a function of proton dose and temperature. These data refer to experiments carried out with the Jet-X CCD12s.

Figure 7. The variation in energy resolution of $6 \mathrm{keV}$ x-rays as a function of proton dose and temperature as predicted from the Jet-X data. The results obtained by applying the physical model described in Section 4 are included.

Figure 8. The variation in energy resolution of $6 \mathrm{keV}$ x-rays as a function of proton dose and temperature. These data show the results of the tests conducted with the EPIC MOS CCD22s. 